\title{
Whole brain radiation therapy does not improve the overall survival of EGFR- mutant NSCLC patients with leptomeningeal metastasis
}

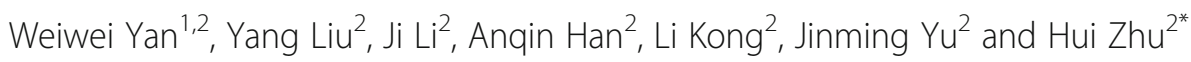

\begin{abstract}
Background: Leptomeningeal metastasis $(L M)$ is a devastating and terminal complication of advanced non-smallcell lung cancer (NSCLC), especially in patients harboring epidermal growth factor receptor (EGFR) mutations. The role of whole brain radiation therapy (WBRT) in the treatment of EGFR-mutant NSCLC patients with LM is not conclusive. Therefore, we conducted a retrospective study to evaluate the therapeutic effect of WBRT in this setting.

Methods: EGFR-mutant NSCLC patients with LM, who had previously received treatment at the Shandong Cancer Hospital and Institute from July 2014 to March 2018 were reviewed retrospectively. LM was diagnosed by positive CSF cytology and/or leptomeningeal-enhanced magnetic resonance imaging (MRI). Survival was estimated using the Kaplan-Meier method.

Results: In total, 51 EGFR-mutated NSCLC patients with LM were eligible for analysis, subdivided into 26 in the WBRT group and 25 in the non-WBRT group. No significant differences were observed in intracranial ORR (15.4\% vs. $16 \%, p=0.952)$ and DCR $(34.7 \%$ vs. $28 \%, p=0.611)$ between the two groups. The median PPFS $_{L M}$ and $O_{L M}$ for the entire cohort were 3.3 months (95\% Cl: 2.77-3.83) and 12.6 months (95\% Cl: 9.66-15.54), respectively. No difference in PFFS $_{L M}$ was observed between the WBRT and non-WBRT groups (median 3.9 vs. 2.8 months; $H R=0.506, p=0.052$ ). The median OS $L M$ was 13.6 months in the WBRT group, compared with 5.7 months in the non-WBRT group (HR= $0.454, p=0.022$ ). Multivariate analyses of $\mathrm{OS}_{\mathrm{LM}}$ showed that $\mathrm{KPS} \geq 80$ at the time of $\mathrm{LM}$ diagnosis $(\mathrm{HR}=0.428,95 \% \mathrm{Cl}$ : $0.19-0.94 ; p=0.034)$ and the administration of EGFR-TKIs ( $H R=0.258,95 \% \mathrm{Cl}: 0.11-0.58 ; p=0.001)$ were independent predictors of survival, but WBRT ( $\mathrm{HR}=0.49,95 \% \mathrm{Cl}: 0.24-1.01 ; p=0.54$ ) was not. Toxicities associated with WBRT or other treatment were rare.
\end{abstract}

Conclusion: For EGFR-mutated NSCLC patients with LM, WBRT did not improve intracranial treatment response and survival statistically.

Keywords: Non-small cell lung cancer, Leptomeningeal metastasis, EGFR mutations, WBRT, Survival, Treatment response

\footnotetext{
* Correspondence: drzhuh@126.com

${ }^{2}$ Department of Radiation Oncology, Shandong Cancer Hospital and Institute,

Shandong First Medical University and Shandong Academy of Medical

Science, Jinan, Shandong, China

Full list of author information is available at the end of the article
}

(c) The Author(s). 2019 Open Access This article is distributed under the terms of the Creative Commons Attribution 4.0 International License (http://creativecommons.org/licenses/by/4.0/), which permits unrestricted use, distribution, and reproduction in any medium, provided you give appropriate credit to the original author(s) and the source, provide a link to the Creative Commons license, and indicate if changes were made. The Creative Commons Public Domain Dedication waiver (http://creativecommons.org/publicdomain/zero/1.0/) applies to the data made available in this article, unless otherwise stated. 


\section{Introduction}

Leptomeningeal metastasis (LM), also termed as neoplastic meningitis, is caused by the diffusion of malignant cells to the leptomeninges and the cerebrospinal fluid (CSF) [1-4]. Approximately 3.8\% of patients with advanced NSCLC have LM at the date of diagnosis or in the course of disease. The incidence of LM in patients harboring epidermal growth factor receptor (EGFR) mutations (9.4\%) is higher than that in patients with wild-type EGFR (1.7\%) [5, 6]. LM often represents a terminal event of NSCLC associated with an extremely poor prognosis, and the median OS of unselected NSCLC patients varies between 3 and 6 months [6-10].

Currently, the treatment for LM consists of using either EGFR-tyrosine kinase inhibitors (TKIs), whole brain radiation therapy (WBRT), Chemotherapy (ChT), intrathecal chemotherapy (ITC), and ventriculoperitonealshunt (VP-shunt) [5, 6, 8, 10-12]. However, the survival benefits of these treatments remain poorly established. The poor permeability of chemotherapeutic or targeted agents through the blood-brain barrier (BBB) may account for the limited role of these treatments. Although WBRT is an effective treatment for patients with multiple brain metastases (BMs), its therapeutic effect in LM patients with EGFR mutations has not been evaluated fully.

Even though most previous studies have been conducted in unselected NSCLC patients, the survival data of LM in EGFR-mutant NSCLC in those studies were in shortage. Therefore, we performed a retrospective analysis of the clinical data of EGFR-mutated NSCLC patients with LM who had received treatment at our hospital from July 2014 to March 2018, aiming to evaluate whether WBRT could provide survival benefits for LM patients with EGFR mutations.

\section{Patients and methods Patients}

Medical records of EGFR-mutant NSCLC patients with cytologically or radiographically confirmed LM treated at the Shandong Cancer Hospital and Institute between July 2014 and March 2018 were collected for this investigation. All patients were diagnosed pathologically with NSCLC. The EGFR status was identified from primary lung tumors using the amplification refractory mutation system (ARMS) analysis. LM diagnosis was based on the detection of malignant cells in the CSF, the focal or diffuse enhancement of leptomeninges, and nerve roots or the ependymal surface on gadolinium-enhanced MRI. The medical ethical committee of the Shandong Cancer Hospital and Institute approved the study protocol.

\section{Clinical data collection}

Data for patient characteristics, tumor features, treatment modalities, and survival outcomes were extracted from medical records. Depending on whether they had received WBRT, enrolled patients were divided into a WBRT group and a non-WBRT group. Patients in the two groups were categorized according to age, gender, smoking status, Karnofsky performance status (KPS) at the time of LM diagnosis, extracranial metastases, NSCLC pathological classification, EGFR mutation status, coexisting BM, and LM-related symptoms and signs at the time of LM diagnosis.

\section{Intracranial response evaluation}

Complete medical histories were obtained, and physical examinations, laboratory examinations, and brain MRI were performed and evaluated before treatment. Lumbar puncture was recommended for the assessment of LM, but it was not mandatory. LM evaluations were performed 1 month after beginning treatment and were followed up once every 2-3 months, or at the time of neurological deterioration. LM response assessment criteria are usually based on published randomized clinical trials, including MRI outcomes, neurological symptoms, and CSF parameters (intracranial pressure; levels of protein, glucose, and chloride; qualitative and quantitative cytology) [13].

In the present study, not all patients were evaluated based on CSF parameters because frequent lumbar puncture was not feasible in some patients. We used MRI outcomes and neurological symptoms to evaluate LM clinical responses. MRI imaging evaluations show subarachnoid masses as measurable lesions and linear or diffuse meningeal enhancement as unmeasurable lesions [13]. The assessment of neurological functions covered cerebral hemisphere symptoms, cranial nerve symptoms, and spinal cord and root symptoms.

A complete response (CR) was defined as the disappearance of all meningeal lesions and neurological symptoms. A partial response (PR) was defined as the improvement in neurological symptoms and a $50 \%$ or more shrinkage in the bidirectional measurement of subarachnoid masses. A stable disease (SD) was defined as stable neurological symptoms and a subarachnoid mass shrinkage of less than $50 \%$ or an increase of less than $25 \%$. One of the following three denoted a progressive disease (PD): a neurological progression, a subarachnoid mass increase of $25 \%$ or more, or an unequivocal progression of existing unmeasurable lesions. The objective response rate (ORR) of intracranial lesions included the combination of $C R$ and $P R$, and the disease control rate (DCR) of intracranial lesions included CR, PR, and SD.

\section{Evaluation of treatment toxicity}

The treatment toxicity associated with WBRT or other treatment were evaluated based on the CTCAE 4.0 edition (Common Terminology Criteria for Adverse Events 
version 4.0) every month, with toxicity graded as mild (grade 1), moderate (grade 2), severe (grade 3) or lifethreatening (grade 4).

\section{Statistical analysis}

All parameters were analyzed as dichotomous variables. Baseline characteristics of patients from the WBRT group and non-WBRT group were compared using the Chisquare or Fisher's exact tests. Intracranial progression-free survival ( $\mathrm{PPFS}_{\mathrm{LM}}$ ) was calculated from the date of $\mathrm{LM}$ diagnosis to the first documentation of intracranial lesion progression or death with documented intracranial progression. Overall survival $\left(\mathrm{OS}_{\mathrm{LM}}\right)$ was calculated from the date of LM diagnosis to death from any cause or last follow-up. The Kaplan-Meier method was used to estimate survival, and the log-rank test was used to compare differences in survival between subgroups. Cox's proportional hazards model was used to evaluate the independent prognostic factors associated with improved survival. All statistically significant variables in the univariate analysis were subjected to the multivariate Cox regression analysis. A $p$-value $<0.05$ was considered to show statistical significance in all analyses. All statistical analyses were performed using SPSS Statistics version 20 (IBM Corporation, NY, USA).

\section{Results}

\section{Clinical characteristics}

Fifty-one EGFR-mutated NSCLC patients with LM were eligible for analysis, subdivided into 26 in the WBRT group and 25 in the non-WBRT group. The baseline characteristics of the two groups are summarized in Table 1. For all baseline characteristics, only EGFR mutation types had statistically different distributions between the two groups $(p<0.05)$. From the entire cohort, 9 (17.6\%) patients had LM at the initial diagnosis of metastatic NSCLC, with the remaining patients were diagnosed with LM during the disease. The median time from the diagnosis of NSCLC to LM was 17.4 months (95\% CI: 8.89-25.92, range 0 to 88.9 months). Twenty patients (39.2\%) had the EGFR exon 19 deletion mutation, and $31(60.8 \%)$ had the exon 21 L858R mutation. All patients showed typical MRI manifestations of LM. Forty $(78.4 \%)$ patients were diagnosed with LM by MRI and CSF cytology, and $11(21.6 \%)$ patients were diagnosed with LM by MRI alone. Forty-four (86.3\%) patients were diagnosed with both LM and BM; 22 of them developed BM before LM, 21 were diagnosed with $\mathrm{BM}$ and LM at the same, and one was diagnosed with BM after LM.

\section{Treatments}

Thirty Gy of WBRT was delivered to patients in the WBRT group in 10 fractions for 5 days per week. Twenty patients received EGFR-TKIs and WBRT, 3 patients underwent WBRT plus ChT, and 3 patients underwent WBRT alone. In the non-WBRT group, 9 patients received EGFR-TKIs alone, 9 patients underwent ChT alone, and 7 patients received both EGFR-TKIs and ChT (Table 2). Among 36 patients received EGFRTKIs, 13 received gefitinib, 19 received erlotinib, and 4 received icotinib.

\section{Intracranial treatment response}

The intracranial treatment responses in the two groups are summarized in Table 3. All patients were evaluated radiologically and clinically after treatment for LM. In the WBRT group, 2 patients (7.69\%) had CR, 2 (7.69\%) had PR, 5 (19.23\%) had SD, and 17 (65.39\%) had PD, whereas, it was $1(4 \%), 3(12 \%), 3(12 \%)$, and $18(72 \%)$ in the non-WBRT group for $\mathrm{CR}, \mathrm{PR}, \mathrm{SD}$, and $\mathrm{PD}$, respectively. Intracranial ORRs were similar between the two groups $(15.4 \%$ vs. $16 \%, p=0.952)$. Intracranial DCR was $34.7 \%$ for the WBRT group and $28 \%$ for the non-WBRT group $(p=0.611)$. No significant differences were observed in intracranial ORR and DCR between the two groups.

\section{Toxicity and safety}

In our study, all 51 patients had good tolerance to the treatment regimen. No patient suspended or interrupted treatment due to serious adverse event. The most frequent acute toxicities associated with WBRT included grade 1 headache in 6 patients, grade 1 nausea/vomiting in 8 patients, and grade 1 dermatitis in 3 patients. No late neurocognitive impairment and reduced quality of life related to WBRT has been detected. The most common adverse reactions of EGFR-TKIs treatment were grade 1 or grade 2 rash, diarrhea, and nausea. Rash occurred in 7 patients, diarrhea in 6 patients, and nausea in 3 patients. The patients had good tolerance to WBRT combined with EGFR-TKIs/ChT and EGFR-TKIs combined with ChT. No patient experienced grade $\geq 3$ treatment-related toxicity in this cohort of patients.

\section{Intracranial progression-free survival}

The median $\mathrm{PFFS}_{\mathrm{LM}}$ for the entire cohort was 3.3 months (95\% CI: 2.77-3.83; Fig. 1a). Intracranial progression was detected in $68.63 \%$ ( 25 of 51 ) patients; $65.39 \%$ (17 of 26 ) had intracranial progression in the WBRT group, while it was $72 \%$ (18 of 25 ) in the non-WBRT group. The difference in $\mathrm{iPFS}_{\mathrm{LM}}$ between the two groups was not statistically significant (median 3.9 vs. 2.8 months; $p=$ 0.052; Fig. 2a). The 36 patients who received EGFR-TKIs demonstrated longer PPFS $_{\mathrm{LM}}$ than those without EGFRTKIs (median 3.9 vs. 2.7 months; $p=0.019$; Fig. $2 \mathrm{~b}$ ). The median $\mathrm{iPFS}_{\mathrm{LM}}$ of patients who underwent EGFR-TKIs and WBRT was similar to that of patients who underwent 
Table 1 Baseline characteristics of enrolled patients

\begin{tabular}{|c|c|c|c|c|}
\hline Characteristic & $\begin{array}{l}\text { Total }(n=51) \\
\mathrm{n}(\%)\end{array}$ & $\begin{array}{l}\text { WBRT }(n=26) \\
\mathrm{n}(\%)\end{array}$ & $\begin{array}{l}\text { non-WBRT }(n=25) \\
\text { n }(\%)\end{array}$ & $P$-value \\
\hline \multicolumn{5}{|c|}{ Age at the time of LM diagnosis (yr.) } \\
\hline Median (range) & $56(32-81)$ & $55(32-81)$ & $56(37-73)$ & \multirow[t]{3}{*}{0.843} \\
\hline$<60$ & $34(66.7)$ & $17(65.4)$ & $17(68)$ & \\
\hline$\geq 60$ & $17(33.3)$ & $9(34.6)$ & $8(32)$ & \\
\hline \multicolumn{5}{|l|}{ Gender } \\
\hline Male & $17(33.3)$ & $8(30.8)$ & $9(36)$ & \multirow[t]{2}{*}{0.692} \\
\hline Female & $34(66.7)$ & $18(69.2)$ & $16(64)$ & \\
\hline \multicolumn{5}{|l|}{ Smoking status } \\
\hline Non-smoker & $40(78.4)$ & $21(80.8)$ & $19(76)$ & \multirow[t]{2}{*}{0.679} \\
\hline Former/current-smoker & $11(21.6)$ & $5(19.2)$ & $6(24)$ & \\
\hline \multicolumn{5}{|c|}{ KPS at the time of LM diagnosis } \\
\hline$\geq 80$ & $39(76.5)$ & $21(80.8)$ & $18(72)$ & \multirow[t]{2}{*}{0.46} \\
\hline$<80$ & $12(23.5)$ & $5(19.2)$ & $7(28)$ & \\
\hline \multicolumn{5}{|l|}{ Pathological classification } \\
\hline Adenocarcinoma & $50(98)$ & $26(100)$ & $24(96)$ & \multirow[t]{2}{*}{0.49} \\
\hline Squamous cell carcinoma & $1(2)$ & $0(0)$ & $1(4)$ & \\
\hline \multicolumn{5}{|l|}{ EGFR mutation status } \\
\hline Exon 19 deletion & $20(39.2)$ & $5(19.2)$ & $15(60)$ & \multirow[t]{2}{*}{0.004} \\
\hline Exon 21 L858R & $31(60.8)$ & $21(80.8)$ & $10(40)$ & \\
\hline \multicolumn{5}{|c|}{ LM present at the initial diagnosis of NSCLC } \\
\hline Yes & $9(17.6)$ & $6(23.1)$ & $3(12)$ & \multirow[t]{2}{*}{0.465} \\
\hline No & $42(82.4)$ & $20(76.9)$ & $22(88)$ & \\
\hline \multicolumn{5}{|c|}{ LM-related Symptoms and signs } \\
\hline Asymptomatic & $12(23.5)$ & $4(15.4)$ & $8(32)$ & \multirow[t]{2}{*}{0.199} \\
\hline Symptomatic & $39(76.5)$ & $22(84.6)$ & $17(68)$ & \\
\hline \multicolumn{5}{|l|}{ The modality of LM diagnosis } \\
\hline MRI+ & $11(21.6)$ & $6(23.1)$ & $5(20)$ & \multirow[t]{2}{*}{0.789} \\
\hline MRI+/cytology+ & $40(78.4)$ & $20(76.9)$ & $20(80)$ & \\
\hline \multicolumn{5}{|l|}{ Co-existing BMs } \\
\hline Yes & $44(86.3)$ & $24(92.3)$ & $20(80)$ & \multirow[t]{2}{*}{0.248} \\
\hline No & $7(13.7)$ & $2(7.7)$ & $5(20)$ & \\
\hline \multicolumn{5}{|c|}{ Extracranial metastases at the time of LM diagnosis } \\
\hline Yes & $37(72.5)$ & $17(65.4)$ & $19(76)$ & \multirow[t]{2}{*}{0.406} \\
\hline No & $14(27.5)$ & $9(34.6)$ & $6(24)$ & \\
\hline
\end{tabular}

LM Leptomeningeal metastasis, KPS Karnofsky performance status, NSCLC Non-small-cell lung cancer, EGFR Epidermal growth factor receptor, MRI Magnetic resonance imaging, $B M s$ Brain metastases

only EGFR-TKIs (median 3.9 vs. 3.2 months; $p=0.379$; Fig. 2c).

In multivariate analyses, KPS $\geq 80$ at the time of LM diagnosis $(\mathrm{HR}=0.338,95 \% \mathrm{CI}: 0.15-0.78 ; p=0.011)$ and the administration of the EGFR-TKI therapy (HR = 0.442, 95\% CI: $0.22-0.91 ; p=0.027$ ) were independent predictors associated with favorable survival. Additional results are detailed in Table 4.

\section{Overall survival}

The last follow-up date was carried out on June 30, 2018. Seventeen patients were still alive by the end of the follow-up. The median $\mathrm{OS}_{\mathrm{LM}}$ for the entire cohort after LM diagnosis was 12.6 months (95\% CI: 9.66-15.54; Fig. 1b). The median $\mathrm{OS}_{\mathrm{LM}}$ were 13.6 and 5.7 months in the WBRT and non-WBRT groups, respectively ( $p=$ 0.022 ; Fig. 3a). The median survival time of the 36 
Table 2 Treatment methods for the enrolled patients

\begin{tabular}{lll}
\hline & WBRT $(n=26)$ & non-WBRT $(n=25)$ \\
\hline WBRT alone & 3 & 0 \\
EGFR-TKIs alone & 0 & 9 \\
ChT alone & 0 & 9 \\
WBRT+EGFR-TKIs & 20 & 0 \\
WBRT+ChT & 3 & 0 \\
EGFR-TKIs+ChT & 0 & 7
\end{tabular}

EGFR Epidermal growth factor receptor, TKI Tyrosine kinase inhibitor, ChT Chemotherapy, WBRT Whole brain radiotherapy

patients who received EGFR-TKIs was 15 months, which was significantly longer than the 4.5 months of the 15 patients who did not receive EGFR-TKIs ( $p=$ 0.002; Fig. 3b). Nineteen patients who received erlotinib achieved significantly longer $\mathrm{OS}_{\mathrm{LM}}$ than those who underwent gefitinib (median $\mathrm{OS}_{\mathrm{LM}} 15$ vs. 6.1 months, $p=0.012$ ). Further analysis showed that the combination of EGFR-TKIs and WBRT did not provide any additional survival benefit, compared to patients who received only EGFR-TKIs (median 13.6 vs. 15 months; $p=0.381$; Fig. 3c).

Results from the variables of KPS, EGFR-TKI therapy, and WBRT subjected to multivariate analysis showed that $\mathrm{KPS} \geq 80$ at the time of $\mathrm{LM}$ diagnosis $(\mathrm{HR}=0.428$, 95\% CI: $0.19-0.94 ; p=0.034)$ and the administration of the EGFR-TKI therapy ( $\mathrm{HR}=0.258,95 \% \mathrm{CI}$ : $0.11-0.58$; $p=0.001$ ) were favorable prognostic factors of $\mathrm{OS}_{\mathrm{LM}}$, whereas, the use of WBRT (HR $=0.49,95 \%$ CI: 0.24-1.01; $p=0.54$ ) was not an independent predictor (Table 5).

\section{Discussion}

This retrospective study is a relatively large cohort of EGFR-mutant LM patients focusing on the role of WBRT in the treatment of LM. The highlight of the study is the homogeneity of all LM patients harboring EGFR mutations. Our findings showed that the administration of EGFR-TKIs and KPS $\geq 80$ at the time of LM diagnosis were associated with prolonged survival.

Table 3 Intracranial Treatment Response for the enrolled patients

\begin{tabular}{lllll}
\hline & All patients $(n=51)$ & WBRT $(n=26)$ & non-WBRT $(n=25)$ & $P$-value \\
\hline CR & $3(5.88 \%)$ & $2(7.69 \%)$ & $1(4 \%)$ & \\
PR & $5(9.8 \%)$ & $2(7.69 \%)$ & $3(12 \%)$ & \\
SD & $8(15.69 \%)$ & $5(19.23 \%)$ & $3(12 \%)$ & \\
PD & $35(68.63 \%)$ & $17(65.39 \%)$ & $18(72 \%)$ & \\
ORR & $8(15.7 \%)$ & $4(15.4 \%)$ & $4(16 \%)$ & 0.952 \\
DCR & $16(31.4 \%)$ & $9(34.7 \%)$ & $7(28 \%)$ & 0.611 \\
\hline
\end{tabular}

$C R$ Complete response, $P R$ Partial response, $S D$ Stable disease, $P D$ Progression disease, ORR Objective response rate, $D C R$ Disease control rate
However, WBRT did not improve intracranial treatment response and survival statistically for patients selected for this study.

The survival benefit and treatment response of WBRT to NSCLC patients with LM remain debatable, especially in patients with EGFR mutations [1, 5, 6, 12, 14-17]. A previous retrospective assessment of 52 EGFR-mutant LM patients showed that WBRT did not provide survival benefit [6]. In another retrospective cohort of 109 LM patients harboring EGFR mutations, patients who underwent WBRT for LM treatment did not achieve longer survival than those without WBRT (9.3 vs. 8.1 months; $p=0.448$ ) [5]. Although Kuiper et al. [17] showed that WBRT could play a role in symptom control, they did not find that it influenced the survival of LM patients harboring EGFR mutations. So far, few retrospective studies have shown that WBRT could bring survival benefits to LM patients with EGFR mutations. Similarly, the survival benefit of WBRT to brain metastases is controversial. A randomized clinical trial QUARTZ showed that except for younger patients, WBRT had no significant effect on survival or quality of life in patients with brain metastases [18].

In LM, a neuraxis disease, CSF circulates dynamically through the whole compartment of the central nervous system (i.e., the intracranial and intraspinal compartments), and malignant cell diffusion affects all CSF compartments [19]. Based on the characteristics of LM, the whole craniospinal axis should be defined as target volume of radiotherapy. However, craniospinal irradiation (CSI) is rarely recommended in clinical work, because of its obvious myelotoxicity and the lack of evidence of its survival benefits $[3,20]$. Subsequently, only the intracranial CSF compartment of the CNS has been irradiated in the treatment of LM with WBRT [17, 19, 21], which could account for the limited role of WBRT.

LM is more common in NSCLC patients harboring EGFR mutations, especially in patients after effective EGFR-TKI treatment [5, 9]. In our study, 23 of 30 developed LM during treatment with EGFR-TKI. After the diagnosis of LM, the 36 patients who received EGFRTKIs achieved significantly longer $\mathrm{iPFS}_{\mathrm{LM}}$ and $\mathrm{OS}_{\mathrm{LM}}$ than patients who did not (median iPFS LM 3.9 vs. 2.7 months, $p=0.019$; median $\mathrm{OS}_{\mathrm{LM}} 10$ vs. 3.3 months, $p=$ 0.002 ), and these findings are consistent with those from previous studies [5, 6, 22, 23]. Li et al. [5] showed that patients who received EGFR-TKIs after LM diagnosis had significantly longer survival compared with those who did not (10.0 vs. 3.3 months; $p<0.001)$. Another retrospective study [6] also reported that EGFR-TKI therapy was an independent predictor of longer survival in 75 EGFR-mutated NSCLC patients with LM. Overall, EGFR-TKIs exhibited good efficacy for EGFR-mutant NSCLC patients with LM. 
$1 \mathrm{~A}$

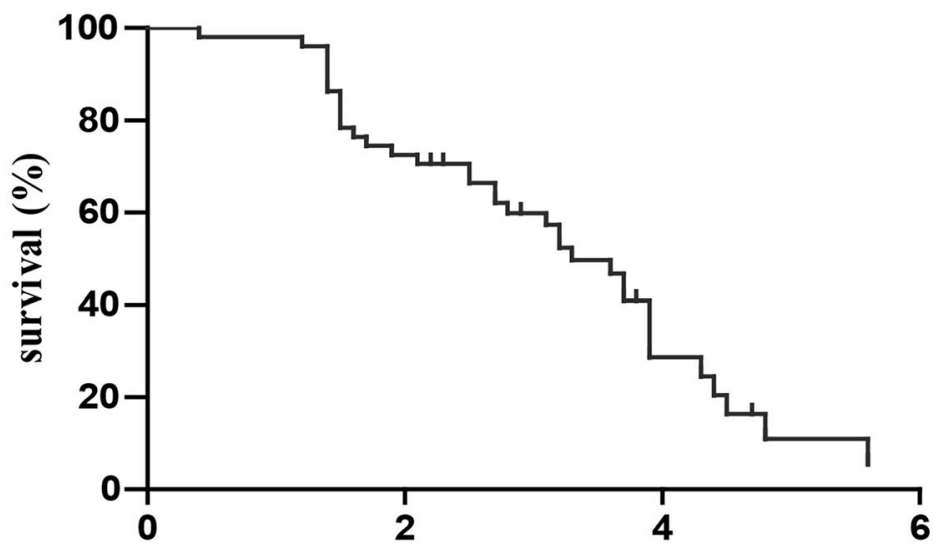

$1 \mathrm{~B}$

i PFS (months)

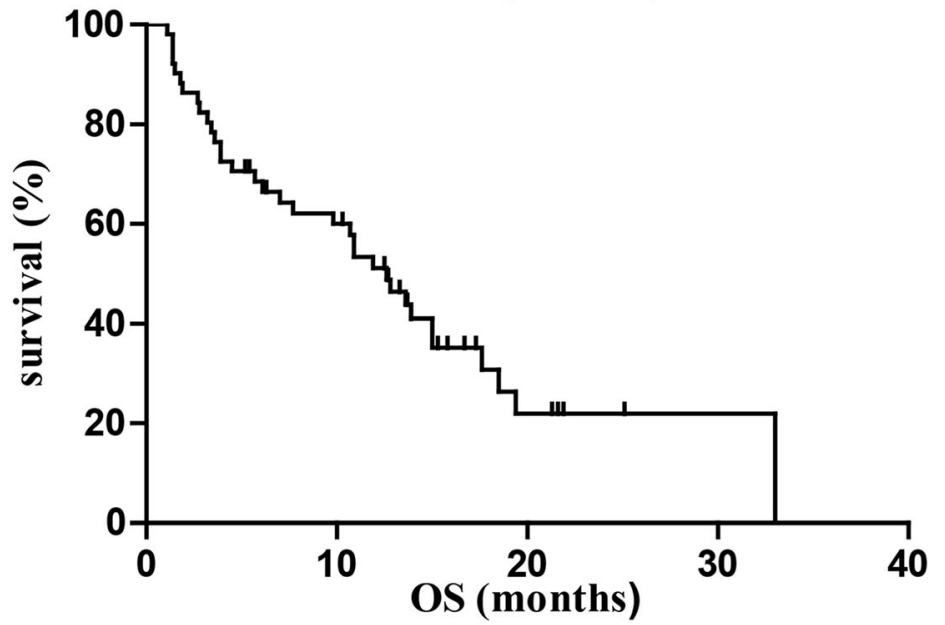

Fig. 1 Intracranial progression-free survival (a) and overall survival (b) after the diagnosis of LM

Currently, there is no sufficient evidence to suggest that the combination of EGFR-TKIs and WBRT has a better survival benefit than EGFR-TKIs alone in EGFRmutant NSCLC patients with LM. However, research on brain metastasis has shown that EGFR-TKIs plus WBRT has a higher response rate and significant improvement in survival, compared with EGFR-TKIs alone in the treatment of BM from EGFR-mutant NSCLC patients [24-27]. Borghetti et al. [28] also confirmed that radiation therapy combined with TKI is a safe and welltolerated therapy for metastatic NSCLC patients with EGFR- or ALK- mutations. In this study, the addition of WBRT to EGFR-TKIs did not lead to any survival benefit in EGFR-mutant LM patients when compared with patients who received only EGFR-TKIs (median OS $_{\mathrm{LM}}$ 13.6 vs. 15 months; $p=0.381$ ). This result is similar to the findings from 33 patients treated with both WBRT and EGFR-TKIs in a previous investigation who did not survive longer than those who received only EGFR-TKIs (median 9.7 vs. 10.1 months; $p=0.778,3$ ). To patients with EGFR-mutant LM, EGFR-TKIs alone could be the better treatment option, with further clinical studies required to certify the hypothesis.

Different EGFR-TKIs options have provided promising outcomes in the treatment of LM form NSCLC. Erlotinib reportedly could be more effective than gefitinib in the treatment of LM in NSCLC patients [29]. Compared to gefitinib, erlotinib has shown higher CSF concentrations (28.7 vs. $3.7 \mathrm{ng} / \mathrm{mL}, p=0.0008)$ and penetration rates ( 2.77 vs. $1.13 \%, p<0.0001)$ [30]. Afatinib and icotinib have also shown efficacy on LM from NSCLC with EGFR mutation [31, 32]. Osimertinib (AZD9291) is a thirdgeneration EGFR-TKI targeting sensitized EGFR mutations and acquired EGFR T790 M resistance mutations [33]. The therapy exhibited a better BBB penetration than the other EGFR-TKIs (gefitinib, rociletinib, or afatinib) [34]. The preliminary results from the phase I BLOOM study (NCT02228369) demonstrated that high-dose osimertinib (160 mg daily) showed encouraging activity and manageable tolerability in pretreated EGFR-mutant NSCLC patients with LM confirmed by CSF cytology [35]. In another study, 20 EGFR-mutant LM patients 


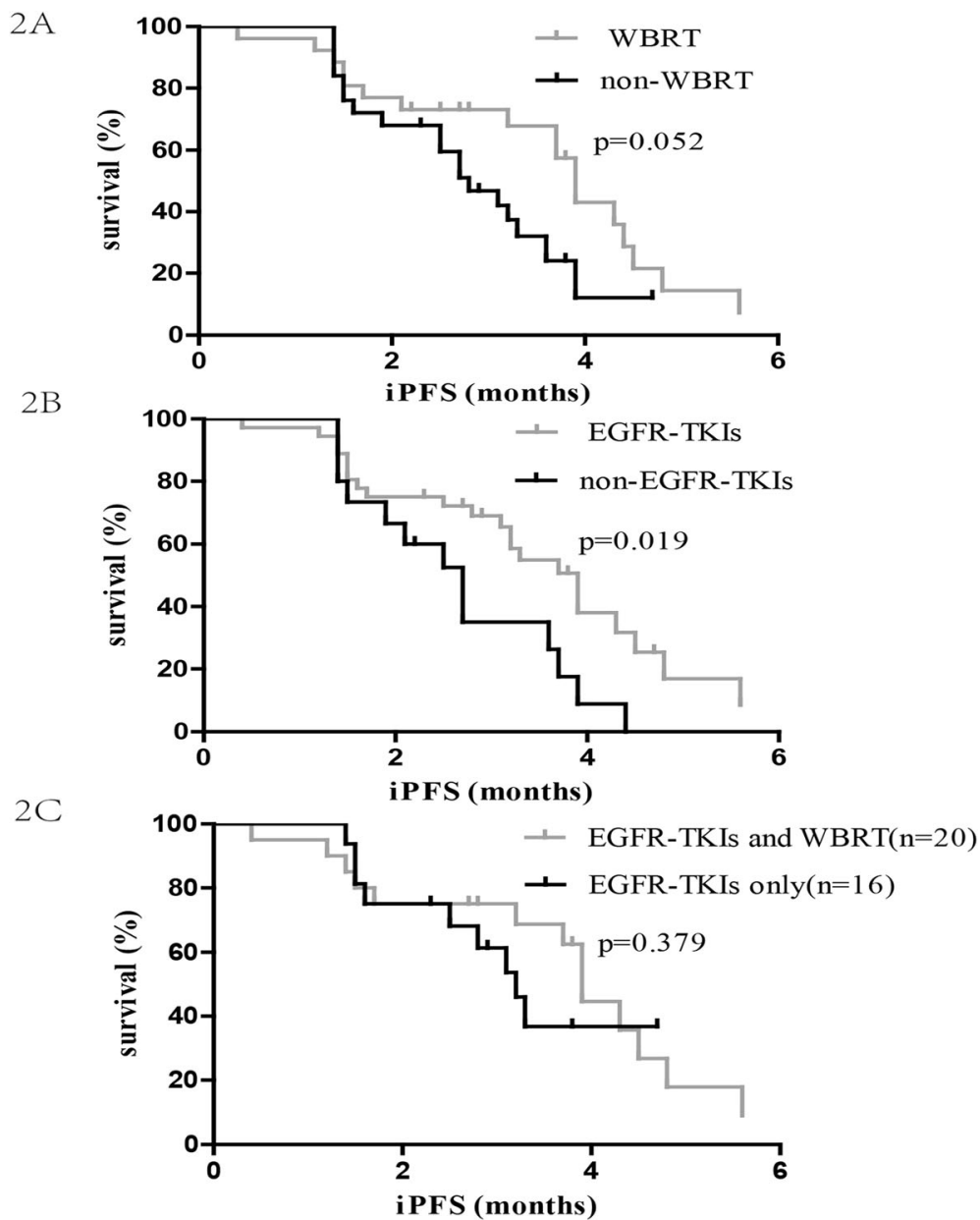

Fig. 2 The Kaplan-Meier analysis showing the intracranial progression-free survival of all patients. a survival of patients who received WBRT compared with those who did not; $\mathbf{b}$ survival of patients who received EGFR-TKls compared with those who did not; c survival of patients who received WBRT plus EGFR-TKIs compared with those who received EGFR-TKIs alone

Table 4 Univariate and multivariate analyses of clinical variables on intracranial progression -free survival

\begin{tabular}{|c|c|c|c|c|}
\hline & $\begin{array}{l}\text { Univariate } \\
\text { HR ( } 95 \% \mathrm{Cl})\end{array}$ & $P$ & $\begin{array}{l}\text { Multivariate } \\
\text { HR ( } 95 \% \mathrm{Cl})\end{array}$ & $P$ \\
\hline Age $(\geq 60 /<60)$ & $1.66(0.78-3.54)$ & 0.17 & & \\
\hline Gender (Female/Male) & $0.64(0.31-1.31)$ & 0.204 & & \\
\hline Smoking status (Smoking/Never) & $1.41(0.65-3.06)$ & 0.375 & & \\
\hline KPS $(\geq 80 /<80)$ & $0.34(0.15-0.78)$ & 0.006 & $0.34(0.15-0.78)$ & 0.011 \\
\hline EGFR mutation (exon 19 deletion/exon 21 L858R) & $0.77(0.39-1.52)$ & 0.43 & & \\
\hline Co-existing BMs (yes/no) & $0.89(0.34-2.33)$ & 0.804 & & \\
\hline Intracranial symptoms (yes/no) & $1.40(0.61-3.22)$ & 0.413 & & \\
\hline LM at the time of NSCLC diagnosis (yes/no) & $0.60(0.21-1.72)$ & 0.324 & & \\
\hline \multicolumn{5}{|l|}{ Treatment for LM } \\
\hline WBRT (yes/no) & $0.51(0.25-1.04)$ & 0.052 & & \\
\hline EGFR-TKIs (yes/no) & $0.45(0.22-0.91)$ & 0.019 & $0.442(0.22-0.91)$ & 0.027 \\
\hline WBRT+EGFR-TKIs/EGFR-TKIs alone & $0.67(0.27-1.67)$ & 0.379 & & \\
\hline
\end{tabular}

LM Leptomeningeal metastasis, KPS Karnofsky performance status, NSCLC Non-small-cell lung cancer, EGFR Epidermal growth factor receptor, TKI Tyrosine kinase inhibitor, WBRT Whole brain radiotherapy, ChT Chemotherapy, BMs Brain metastases 
$3 \mathrm{~A}$

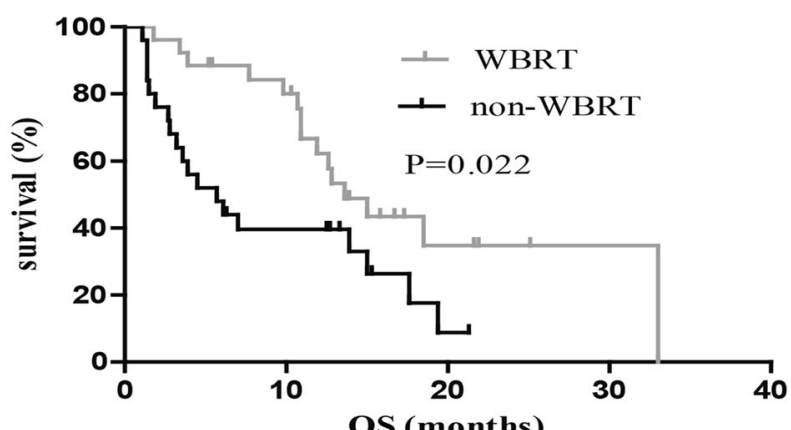

$3 B$

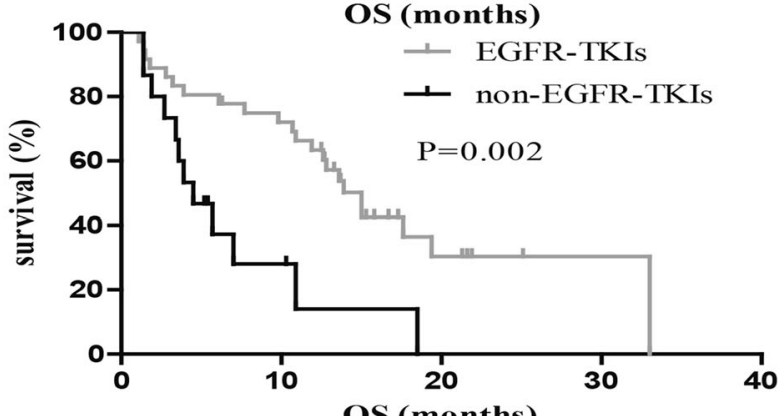

$3 \mathrm{C}$

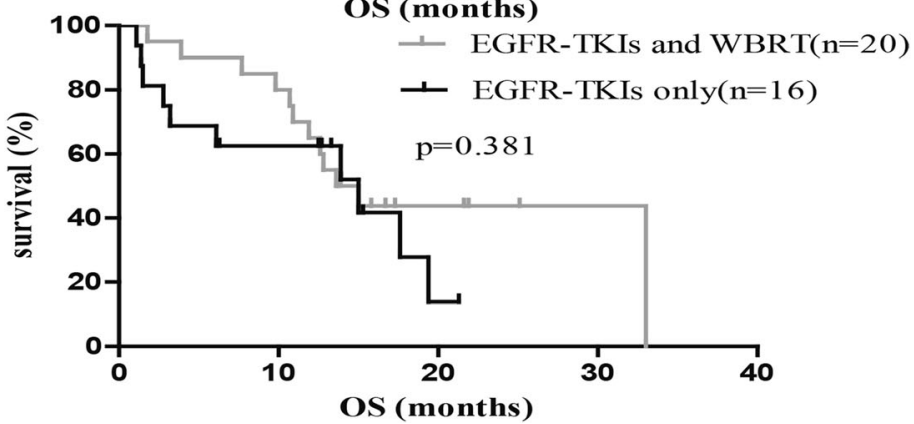

Fig. 3 The Kaplan-Meier analysis showing the overall survival of all patients. a survival of patients who received WBRT compared with those who did not; $\mathbf{b}$ survival of patients who received EGFR-TKIs compared with those who did not; c survival of patients who received WBRT plus EGFRTKIs compared with those who received EGFR-TKIs alone

Table 5 Univariate and multivariate analyses of clinical variables on overall survival

\begin{tabular}{llll}
\hline & Univariate & $P$ & Multivariate \\
HR (95\% Cl)
\end{tabular}

LM Leptomeningeal metastasis, KPS Karnofsky performance status, NSCLC Non-small-cell lung cancer, EGFR Epidermal growth factor receptor, TKI Tyrosine kinase inhibitor, WBRT Whole brain radiotherapy, ChT Chemotherapy, BMs Brain metastases 
treated with osimertinib had an OS of 18.0 months [36], whereas, OS was only 3.1 months in a cohort of 32 LM patients treated with first- and second-generation EGFR-TKIs [16]. Osimertinib appears to be more effective than the first- and second-generation EGFRTKIs. However, the use of osimertinib in the treatment of EGFR-mutant LM has not been approved yet, and further randomized clinical studies are needed to confirm its prowess.

This study, despite its mixed findings, also has several limitations. Firstly, the retrospective design and small sample size affected its statistical power. Moreover, the data were obtained retrospectively from medical records, and there may have been biasing in patient selection. Secondly, the intracranial therapeutic response was not evaluated using CSF parameters. Therefore, our conclusions should be interpreted cautiously.

\section{Conclusion}

In conclusion, our study revealed that EGFR-TKIs seems to have an excellent control of LM in NSCLC patients with EGFR mutations even if WBRT is not delivered; on the other hand, WBRT seems to positively influence the survival of these patients even if it does not get the statistical significance. Probably, WBRT could be omitted in asymptomatic patients when EGFR-TKIs are available for LM, thus further avoiding the side effects of WBRT.

\begin{abstract}
Abbreviations
ARMS: Amplification refractory mutation system; BBB: Blood-brain barrier; BMs: Brain metastases; ChT: Chemotherapy; CR: Complete response; CSF: Cerebrospinal fluid; DCR: Disease control rate; EGFR: Epidermal growth factor receptor; iPFS: Intracranial progression-free survival; ITC: Intrathecal chemotherapy; KPS: Karnofsky performance status; LM: Leptomeningeal metastasis; MRI: Magnetic Resonance Imaging; NSCLC: Non-small cell lung cancer; ORR: Objective response rate; OS: Overall survival; PD: Progressive disease; PR: Partial response; SD: Stable disease; TKI: Tyrosine kinase inhibitor; VP-shunt: Ventriculoperitoneal-shunt; WBRT: Whole brain radiation therapy
\end{abstract}

\section{Acknowledgments}

Not applicable.

\section{Authors' contributions}

$W Y$ and $H Z$ conceived and drafted the manuscript. $Y L, J L$ and $A H$ acquired and analyzed the patient data. LK and JY edited and corrected the manuscript. All authors read and approved the final manuscript.

\section{Funding}

This work was supported by the National Key Research and Development Program (No.2018YFC1313201).

\section{Availability of data and materials}

All data and materials can be found in "Patient, materials and methods" section within the article.

\section{Ethics approval and consent to participate}

The study was approved by the institutional review board and ethics committee of the Shandong Cancer Hospital and Institute. All the patients enrolled in this study signed informed consents.

\section{Consent for publication}

Not application.

\section{Competing interests}

The authors declare that they have no competing interests.

\section{Author details}

${ }^{1}$ School of Medicine and Life Sciences, University of Jinan-Shandong Academy of Medical Sciences, Jinan, Shandong, China. ${ }^{2}$ Department of Radiation Oncology, Shandong Cancer Hospital and Institute, Shandong First Medical University and Shandong Academy of Medical Science, Jinan, Shandong, China.

Received: 29 June 2019 Accepted: 9 September 2019 Published online: 14 September 2019

\section{References}

1. Remon J, Le Rhun E, Besse B. Leptomeningeal carcinomatosis in non-small cell lung cancer patients: a continuing challenge in the personalized treatment era. Cancer Treat Rev. 2017:53:128-37.

2. Cheng $H$, Perez-Soler R. Leptomeningeal metastases in non-small-cell lung cancer. Lancet Oncol. 2018;19(1):e43-55.

3. Chamberlain MC. Leptomeningeal metastasis. Curr Opin Oncol. 2010;22(6): 627-35.

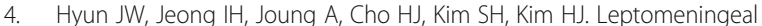
metastasis: clinical experience of 519 cases. Eur J Cancer. 2016;56:107-14.

5. Li YS, Jiang BY, Yang JJ, Tu HY, Zhou Q, Guo WB, et al. Leptomeningeal metastases in patients with NSCLC with EGFR mutations. J Thorac Oncol. 2016:11(11):1962-9.

6. Liao BC, Lee JH, Lin CC, Chen YF, Chang CH, Ho CC, et al. Epidermal growth factor receptor tyrosine kinase inhibitors for non-small-cell lung cancer patients with leptomeningeal carcinomatosis. J Thorac Oncol. 2015;10(12): 1754-61.

7. Lee Y, Han JY, Kim HT, Yun T, Lee GK, Kim HY, et al. Impact of EGFR tyrosine kinase inhibitors versus chemotherapy on the development of leptomeningeal metastasis in never smokers with advanced adenocarcinoma of the lung. J Neuro-Oncol. 2013;115(1):95-101.

8. Lee SJ, Lee JI, Nam DH, Ahn YC, Han JH, Sun JM, et al. Leptomeningeal carcinomatosis in non-small-cell lung cancer patients: impact on survival and correlated prognostic factors. J Thorac Oncol. 2013:8(2):185-91.

9. Wu YL, Zhao Q, Deng L, Zhang Y, Zhou XJ, Li YY, et al. Leptomeningeal metastasis after effective first-generation EGFR TKI treatment of advanced non-small cell lung cancer. Lung Cancer. 2019;127:1-5.

10. Riess JW, Nagpal S, Iv M, Zeineh M, Gubens MA, Ramchandran K, et al. Prolonged survival of patients with non-small-cell lung cancer with leptomeningeal carcinomatosis in the modern treatment era. Clin Lung Cancer. 2014;15(3):202-6.

11. Umemura S, Tsubouchi K, Yoshioka H, Hotta K, Takigawa N, Fujiwara K, et al. Clinical outcome in patients with leptomeningeal metastasis from non-small cell lung cancer: Okayama lung cancer study group. Lung Cancer. 2012; 77(1):134-9.

12. Morris PG, Reiner AS, Szenberg OR, Clarke $J L$, Panageas KS, Perez HR, et al. Leptomeningeal metastasis from non-small cell lung cancer: survival and the impact of whole brain radiotherapy. J Thorac Oncol. 2012;7(2):382-5.

13. Xu Y, Hu M, Zhang M, Zhong W, Yin X, Sun Y, et al. Prospective study revealed prognostic significance of responses in leptomeningeal metastasis and clinical value of cerebrospinal fluid-based liquid biopsy. Lung Cancer. 2018;125:142-9.

14. Gwak HS, Joo J, Kim S, Yoo H, Shin SH, Han JY, et al. Analysis of treatment outcomes of intraventricular chemotherapy in 105 patients for leptomeningeal carcinomatosis from non-small-cell lung cancer. J Thorac Oncol. 2013:8(5):599-605.

15. Park JH, Kim YJ, Lee JO, Lee KW, Kim JH, Bang SM, et al. Clinical outcomes of leptomeningeal metastasis in patients with non-small cell lung cancer in the modern chemotherapy era. Lung Cancer. 2012;76(3):387-92.

16. Kuiper $\mathrm{L}$, Bahce I, Voorhoeve C, Yaqub M, Heideman DA, Thunnissen E, et al. Detecting resistance in EGFR-mutated non-small-cell lung cancer after clonal selection through targeted therapy. Pers Med. 2015;12(2):63-6.

17. Kuiper JL, Hendriks LE, van der Wekken AJ, de Langen AJ, Bahce I, Thunnissen E, et al. Treatment and survival of patients with EGFR-mutated non-small cell lung cancer and leptomeningeal metastasis: a retrospective cohort analysis. Lung Cancer. 2015;89(3):255-61.

18. Mulvenna P, Nankivell M, Barton R, Faivre-Finn C, Wilson P, McColl E, et al. Dexamethasone and supportive care with or without whole brain 
radiotherapy in treating patients with non-small cell lung cancer with brain metastases unsuitable for resection or stereotactic radiotherapy (QUARTZ): results from a phase 3, non-inferiority, randomised trial. Lancet. 2016; 388(10055):2004-14

19. Sakaguchi M, Maebayashi T, Aizawa T, Ishibashi N, Saito T. Patient outcomes of whole brain radiotherapy for brain metastases versus leptomeningeal metastases: a retrospective study. Asia Pac J Clin Oncol. 2017;13(5):e449-e57.

20. Chamberlain MC. Neoplastic meningitis. Oncologist. 2008;13(9):967-77.

21. Chamberlain MC, Eaton K. Is there a role for whole brain radiotherapy in the treatment of leptomeningeal metastases? J Thorac Oncol. 2012;7(7):1204; author reply -5 .

22. Yi HG, Kim HJ, Kim YJ, Han SW, Oh DY, Lee SH, et al. Epidermal growth factor receptor (EGFR) tyrosine kinase inhibitors (TKIs) are effective for leptomeningeal metastasis from non-small cell lung cancer patients with sensitive EGFR mutation or other predictive factors of good response for EGFR TKI. Lung Cancer. 2009;65(1):80-4.

23. So T, Inoue M, Chikaishi Y, Nose N, Sugio K, Yasumoto K. Gefitinib and a ventriculo-peritoneal shunt to manage carcinomatous meningitis from nonsmall-cell lung cancer: report of two cases. Surg Today. 2009;39(7):598-602.

24. Chen Y, Yang J, Li X, Hao D, Wu X, Yang Y, et al. First-line epidermal growth factor receptor (EGFR)-tyrosine kinase inhibitor alone or with whole-brain radiotherapy for brain metastases in patients with EGFR-mutated lung adenocarcinoma. Cancer Sci. 2016;107(12):1800-5.

25. Lu Y, Fan Y. Combined action of EGFR tyrosine kinase inhibitors and wholebrain radiotherapy on EGFR-mutated non-small-cell lung cancer patients with brain metastasis. Onco Targets Ther. 2016;9:1135-43.

26. Zeng YD, Zhang L, Liao H, Liang Y, Xu F, Liu JL, et al. Gefitinib alone or with concomitant whole brain radiotherapy for patients with brain metastasis from non-small-cell lung cancer: a retrospective study. Asian Pac J Cancer Prev. 2012;13(3):909-14.

27. Zhou L, He J, Xiong W, Liu Y, Xiang J, Yu Q, et al. Impact of whole brain radiation therapy on CSF penetration ability of Icotinib in EGFR-mutated non-small cell lung cancer patients with brain metastases: results of phase I dose-escalation study. Lung Cancer. 2016;96:93-100.

28. Borghetti P, Bonu ML, Giubbolini R, Levra NG, Mazzola R, Perna M, et al. Concomitant radiotherapy and TKI in metastatic EGFR- or ALK-mutated nonsmall cell lung cancer: a multicentric analysis on behalf of AIRO lung cancer study group. Radiol Med. 2019;124(7):662-70.

29. Lee E, Keam B, Kim DW, Kim TM, Lee SH, Chung DH, et al. Erlotinib versus gefitinib for control of leptomeningeal carcinomatosis in non-small-cell lung cancer. J Thorac Oncol. 2013;8(8):1069-74.

30. Togashi Y, Masago K, Masuda S, Mizuno T, Fukudo M, Ikemi Y, et al. Cerebrospinal fluid concentration of gefitinib and erlotinib in patients with non-small cell lung cancer. Cancer Chemother Pharmacol. 2012;70(3):399-405.

31. Tamiya A, Tamiya M, Nishihara T, Shiroyama T, Nakao K, Tsuji T, et al. Cerebrospinal fluid penetration rate and efficacy of Afatinib in patients with EGFR mutation-positive non-small cell lung cancer with leptomeningeal carcinomatosis: a multicenter prospective study. Anticancer Res. 2017;37(8): 4177-82.

32. Gong L, Xiong M, Huang Z, Miao L, Fan Y. Icotinib might be effective for the treatment of leptomeningeal carcinomatosis in non-small cell lung cancer with sensitive EGFR mutations. Lung Cancer. 2015;89(3):268-73.

33. Tan CS, Cho BC, Soo RA. Next-generation epidermal growth factor receptor tyrosine kinase inhibitors in epidermal growth factor receptor -mutant nonsmall cell lung cancer. Lung Cancer. 2016;93:59-68.

34. Ballard P, Yates JW, Yang Z, Kim DW, Yang JC, Cantarini M, et al. Preclinical comparison of Osimertinib with other EGFR-TKIs in EGFR-mutant NSCLC brain metastases models, and early evidence of clinical brain metastases activity. Clin Cancer Res. 2016;22(20):5130-40.

35. Yang JC-H, Cho BC, Kim D-W, Kim S-W, Lee J-S, Su W-C, et al. Osimertinib for patients (pts) with leptomeningeal metastases (LM) from EGFR-mutant non-small cell lung cancer (NSCLC): updated results from the BLOOM study. J Clin Oncol. 2017;35(15_suppl):2020.

36. Saboundji K, Auliac JB, Perol M, Francois G, Janicot H, Marcq M, et al. Efficacy of Osimertinib in EGFR-mutated non-small cell lung cancer with leptomeningeal metastases pretreated with EGFR-tyrosine kinase inhibitors. Target Oncol. 2018;13(4):501-7.

\section{Publisher's Note}

Springer Nature remains neutral with regard to jurisdictional claims in published maps and institutional affiliations.

\section{Ready to submit your research? Choose BMC and benefit from}

- fast, convenient online submission

- thorough peer review by experienced researchers in your field

- rapid publication on acceptance

- support for research data, including large and complex data types

- gold Open Access which fosters wider collaboration and increased citations

- maximum visibility for your research: over $100 \mathrm{M}$ website views per year

At BMC, research is always in progress.

Learn more biomedcentral.com/submissions 